\title{
Ovarian immature teratoma grade II in pregnancy, two cases report and literature review
}

\author{
Fatemeh Homaei Shandiz ${ }^{1}$ and Ali Emadi Torghabeh ${ }^{1}$ \\ ${ }^{1}$ Mashhad University of Medical Sciences
}

February 27, 2021

\begin{abstract}
Ovarian immature teratoma is a rare tumor during pregnancy. In this disease, the role of adjuvant chemotherapy for initial stage 1, grade 2 and 3 is essential in pregnancy. Two cases of ovarian immature teratoma grade 2 in pregnancy with two different managements and outcomes have been illustrated down below.
\end{abstract}

Title: Ovarian immature teratoma grade II in pregnancy, two cases report and literature review Running title: Ovarian immature teratoma in pregnancy

Fatemeh Homaei Shandiz: 1) Cancer Research Center, Mashhad University of Medical Sciences, Mashhad, Iran and 2) Department of Radiotherapy and Oncology, Faculty of Medicine, Mashhad University of Medical Sciences, Mashhad, Iran. Email: HomaeeF@mums.ac.ir ORCID: 0000-0002-7718-5387

Ali Emadi Torghabeh: 1) Cancer Research Center, Mashhad University of Medical Sciences, Mashhad, Iran and 2) Department of Radiotherapy and Oncology, Faculty of Medicine, Mashhad University of Medical Sciences, Mashhad, Iran. Email: EmadiTA@mums.ac.ir ORCID: 0000-0002-8853-8635

Corresponding author: Ali Emadi Torghabeh. Omid Hospital, Koohsangi Street, Mashhad, 9176613775 Iran.

Tell: 00985138426082. Fax: 00985138461518. Email: EmadiTA@mums.ac.ir

Abstract:

Ovarian immature teratoma is a rare tumor during pregnancy. In this disease, the role of adjuvant chemotherapy for initial stage 1 , grade 2 and 3 is essential in pregnancy. Two cases of ovarian immature teratoma grade 2 in pregnancy with two different managements and outcomes have been illustrated down below.

Keywords: chemotherapy, ovarian immature teratoma grade 2, pregnancy

Introduction:

On one hand the risk of malignant ovarian tumor in pregnancy is relatively low. On the other hand, ovarian germ cell tumors are rare. Nonetheless, they are curable in all stages [1]. Pure ovarian immature teratoma is a term that describes the germ cell tumor of the ovary by excluding patterns of endodermal sinus tumor, choriocarcinoma, and dysgerminoma [2]. Ovarian immature teratoma is a malignant germ cell tumor and represents less than $1 \%$ of ovarian malignant tumors; tissues are derived from the three germ layers (endo-, meso- and ectoderm). Tumor grading is based on the amount of immature neuroepithelium present and the prognosis is directly correlated to histological grade. Its rapid growth leads to large tumors and an early diagnosis [3]. Pregnancy complicated by an ovarian immature teratoma is fairly rare, with a reported 
incidence of $0.07 \%$ [4]. Treatment of this tumor in pregnancy is mainly by surgery, especially in the second pregnancy trimester and then systemic chemotherapy may be essential base on the tumor grade and disease stage. In this article, we report two cases of grade 2 ovarian immature teratoma in pregnancy that referred to our clinic at a same time which is rare.

Case 1:

The patient was a primigravid 21 year old woman whose complaint was abdominal pain in sixth week of pregnancy. After history taking and physical examination, the patient underwent an abdomen-pelvic ultrasonography that reported an alive embryo at age of 6-7 weeks together with a right ovarian cyst at the size of $140 * 150 \mathrm{~mm}$ consist of solid component. The patient underwent laparotomy and right ovarian cystectomy on the thirty-first of July, 2016. Pathologic assessment of ovarian tumor revealed;" Immature teratoma, Grade 2" ;( Fig 1). The patient referred and evaluated in a multidisciplinary team (MDT) on 19th of September 2016. MDT recommendations were to apply chest x- ray (CXR) by shielding of abdomen and pelvis, chemotherapy by BEP regimen (bleomycin + etoposide + cisplatin) with continued pregnancy. At delivery time, if Cesarean section $(\mathrm{C} / \mathrm{S})$ indicated, complete surgical staging accomplishes concurrently and if normal vaginal delivery(NVD) indicated, complete surgical staging by fertility preserving approach accomplish later. Eventual chemotherapy complications explained to the patient and accept it. Subsequently, she signed the informed consent.

Results of pre- chemotherapy evaluation was normal.

Chemotherapy by BEP regimen began every 3 week whereas the age of pregnancy was 14 weeks +6 days. The patient was also under vision of an obstetrics gynecologist during treatment. After completing of 3 cycles of chemotherapy, whereas the age of fetus was 26 weeks, the patient affected by blood hypertension followed by eclampsia and underwent $\mathrm{C} / \mathrm{S}$, complete right oophorectomy and peritoneal fluid sampling on twenty-first of December 2016. Pathologic assessment of right ovary and peritoneal fluid was negative for malignancy. General condition of the patient was good but unfortunately, male newborn died. Thereafter the patient followed in our clinic every 2- 3 months. Now, after about 2 years of C/S, all clinic and paraclinic evaluations including tumor markers, imaging of chest and abdomen-pelvic are normal. Now, she has a one year old baby.

Case 2:

The patient was a multi gravid 2, live 1, 29 year old woman whose complaint was abdominal pain in second month of pregnancy. After history taking and physical examination, she underwent an abdomen-pelvic ultrasonography that reported an alive embryo at age of 7 weeks together with a left adnexal cystic mass at the size of $82 * 64 \mathrm{~mm}$ consist of a $58^{*} 41 \mathrm{~mm}$ solid component.( Fig 2) The patient didn't pursue until one month later that she underwent abdomen-pelvic ultrasonography again. This time, sonologist reported a mixed mass measuring $146^{*} 140^{*} 96 \mathrm{~mm}$ with a solid component measuring $115^{*} 97^{*} 83 \mathrm{~mm}$ in the left adnex. The patient underwent laparascopic resection of the left ovarian mass on September 4th, 2016. Pathologic assessment of ovarian tumor revealed;" Immature teratoma, Grade 2". The patient was referred and evaluated in a MDT of our clinic on 19th of September 2016. MDT recommended applying CXR by shielding of abdomen and pelvis, chemotherapy by BEP regimen and completion of pregnancy. At delivery time, if $\mathrm{C} / \mathrm{S}$ indicated, complete surgical staging accomplishes concurrently and if NVD indicated, complete surgical staging accomplish later.

In another ultrasonography at this time, the fetal age was 17 weeks and there was some ascites in the abdominal cavity. CXR was normal at that time. Eventual chemotherapy complications explained to the patient. Nevertheless, she refused coming to our clinic and doing chemotherapy until she accomplished her pregnancy. At the delivery time, she underwent $\mathrm{C} / \mathrm{S}$, resection of abdominal mass on twenty-first of February 2017 and a male baby born. Surgeon reported multiple mass in the bowel meso and omentum along with hemorrhagic ascites. Pathologic assessment of the resected mass revealed;" metastatic immature teratoma, grade 2 with positive ascites fluid for malignancy". 10 days after surgery, laboratory evaluations were: 


\section{CBC\&KFT\&LFT: OK AFP=117 U/ml BHCG=0 Miu/ml CA125=146 U/ml}

The patient advised for BEP regimen chemotherapy and she received this treatment by 21 days intervals beginning from 19th March 2017. On twenty-second May 2017 after 3 course of chemotherapy, paraclinic assessments report $\mathrm{AFP}=7.3 \mathrm{U} / \mathrm{ml}$ and $\mathrm{CA} 125=14 \mathrm{U} / \mathrm{ml}$. Abdomen- pelvic CT scan showed peritoneal seeding and implants around the liver and in sub diaphragmatic, paracolic and pelvic regions and also two bilateral supra diaphragmatic lymphadenopathy(LAP). Then chemotherapy courses 4 and 5 administered by deleting of bleomycin. After 5 courses of BEP paraclinic evaluations on 29th of July 2017 revealed:

$\mathrm{AFP}=4.9 \mathrm{U} / \mathrm{ml} \mathrm{CA} 125=19.9 \mathrm{U} / \mathrm{ml} \mathrm{LDH}=232 \mathrm{U} / \mathrm{L}$

Thoracic CT scan: right supradiaphragmatic LAP. Abdomen- pelvic CT scan: relatively large peritoneal implants around the liver, in the right paracolic and pelvis regions that become greater than previous CT scan and showed radiologic disease progression. Based on MDT recommendation, the patient underwent needle biopsy from supra diaphragmatic mass at 16 August 2017. Its pathologic assessment showed; "mature teratoma"; (Fig 3). Given the low tumor marker and CT scan report, surgical laparotomy recommended by MDT and the patient underwent surgical resection of the masses around liver and ileal meso masses on 18th of September 2017 but the surgeon report diffuse tumoral seeding in abdominal wall. Pathologic report of all of resected mass was;"immature teratoma". Thereafter the patient followed up.

Another CT scan on November 7th, 2017 revealed a round soft tissue mass in anterior part of right lung in the right paracardiac region and irregular hypoechoic liver foci in the posterior segment of right lobe near hepatic capsule due to previous surgery with some fluid around the hepatic capsule and mild splenomegaly. Lab tests on twenty-first of October 2017 showed: $A F P=2 \mathrm{U} / \mathrm{ml} \mathrm{BHCG}=0 \mathrm{Miu} / \mathrm{ml}$. The patient discussed in MDT meeting and advised for surgical resection of intrathoracic lesions. She underwent this surgery on 16th of December 2017. Pathologic evaluation of these lesions was; "immature teratoma". After a month of the surgery, CT scan showed pulmonary nodules add ascites and in lab tests, AFP $=2 \mathrm{U} / \mathrm{ml}$ on 23th of January 2018. MDT recommended chemotherapy by regimen paclitaxel $\left(175 \mathrm{mg} / \mathrm{m}^{2}\right)+$ carboplatin $(\mathrm{AUC}=5)$ every 3 weeks in this situation and she treated by this regimen for 6 cycles till 12th of July 2018. Treatment of the patient terminated in July 2018 and thereafter she followed up. At this time, she is asymptomatic and has a stable disease.

\section{Discussion:}

Pure immature teratomas, first characterized by Norris et al in 1976, are rare germ cell tumors which involve three germ layers with at least one of the components having an immature appearance $[2,5]$. Survival is determined by the size and stage of teratomas, but the grade of the primary tumor is the most important determinant of the likelihood of extra ovarian spread and for the subsequent course. Grading is based on the amount of immature neural tissue [2]. Zhang R. et al reported 5-year survival rate as $92 . \%$ for immature teratoma based on 63 (non-pregnant) cases with immature teratoma among 145 malignant ovarian germ cell tumor cases and histology, surgical approach, chemotherapy and regimens were not predictive of five-year survival rates. Moreover, they concluded that fertility-sparing treatment should be considered for ovarian germ cell tumors regardless of the FIGO stage [6].

Young et al. showed that prognosis for ovarian malignancies was not complicated by a concurrent gestation if adequate treatment is administered timely [7]. Management of ovarian tumors in pregnancy requires a multidisciplinary approach and therapeutic decision should take into account histology, grade and stage of the tumor, and the pregnancy age [8]. Prognosis for immature teratomas has improved due to the routine use of imaging during pregnancy and because of chemotherapy and most ovarian cancers associated with pregnancy are detected by ultrasonography [9]. The main presenting symptom for ovarian immature teratomas during pregnancy is adnexal mass followed by abdominal or pelvic pain. Some patients present with combined abdominal pain and mass and some patients are asymptomatic and detect by routine ultrasonography and by elevated serum AFP [10]. Both our patients also refer with primary complaint of abdominal pain. Given malignant ovarian germ cell tumors are usually unilateral, except advanced stage cases with metastasis to the contralateral ovary, unilateral salpingo oopherectomy with preservation of the contralateral ovary 
and uterus are appropriate for treatment of most cases. If metastatic disease is detected during surgery, cytoreductive surgery is recommended. Second look laparotomy for germ cell tumors is controversial and if inadequate staging was present at the first operation, second look surgery or CT should be considered [11]. The poor prognosis of malignant germ cell tumors treated by surgery alone indicates a need for adjuvant chemotherapy [4]. The risk of major malformation during the first trimester of pregnancy is $10 \%$ for single agent chemotherapy and $25 \%$ for combination chemotherapy [5]. Afterwards, the second trimester seems safer for chemotherapy.

Based on NCCN guideline; patients affected by stage 1, grade 1 ovarian immature teratoma have excellent prognosis without adjuvant chemotherapy, therefore these patients only observe postoperatively. The patients with stage 1 and grade 2 or 3 of immature teratoma and the patients with stages 2 or 3 at any grades need for postoperative chemotherapy by BEP regimen for 3- 4 cycles every 3 weeks. After completion of chemotherapy, if you have complete clinical responses in lab and imaging studies, observation is recommended. But if the imaging studies reveal residual disease with normal tumor marker level, both surgery and observation are advisable and ultimately, if the tumor marker level is high and the imaging studies reveal residual disease too, salvage or high dose chemotherapy are recommended.

Although there is limited experience for using this regimen during pregnancy $[12,13]$, the BEP treatment has been associated with ventriculomegaly, transient neonatal neutropenia and bilateral sensorineural hearing loss [14]. Whether blood hypertension and eclampsia associate with BEP treatment, more data are mandatory for safety profile detection of this regimen.

Conclusion:

Based on our two mentioned cases and the other data, it seems that postoperative chemotherapy during pregnancy after first trimester is essential for patents with initial disease stage 1, grade 2 ovarian immature teratoma and it associates with lower disease progression and recurrence.

Acknowledgement: Tanks to S.T.Jamshidi and A.Ziaolhagh for their assistance in preparing pathologic reports and figures. Tanks to Mahla Babaei for English editing.

References:

1. Patterson DM, Rustin GJ. Controversies in the management of germ cell tumours of the ovary. Curr Opin Oncol 2006 Sep; 18 (5): 500-6.

2. Norris HJ, Zirkin HJ, Benson WL. Immature (malignant) teratoma of the ovary: a clinical and pathologic study of 58 cases. Cancer 1976; 37: 2359-72.

3. Noun M, Ennachit M, Boufettal H, Elmouatacim K, Samouh N. The ovarian immature teratoma with gliomatosisperitonei. J Gynecol Obstet Biol Reprod (Paris) 2007 Oct; 36 (6): 595-601.

4. Christman JE, Teng NN, Lebovic GS, Sikic BI. Delivery of a normal infant following cisplatin, vinblastine, and bleomycin (PVB) chemotherapy for malignant teratoma of the ovary during pregnancy. Gynecol Oncol 1990; 37: 292-5.

5. Mourali M, Ben Haj Hassine A, El Fekih C, Essoussi-Chikhaoui J, Binous N, Ben Zineb N, et al. Immature teratoma of the ovary and pregnancy. (In French). Tunis Med 2010; 88: 507-12.

6. Helage S, Borghese B, Depinay C, Alexandre J, Chopin N. Ovarian immature teratoma during pregnancy. (In French). Presse Medicale 2011; 40: 102-105.

7. Clinkard DJ, Khalifa M, Osborned RJ, Bouffet E. Successful management of medulloblastoma arising in an immature ovarian teratoma in pregnancy. Gynecol Oncol 2011; 120: 311-2.

8. Mendivil AA, Brown III JV, Abaid LN, Rettenmaier MA, Micha JP, Wabe MA, et al. Robotic-assisted surgery for the treatment of pelvic masses in pregnant patients: A series of four cases and literature review. Journal of Robotic Surgery 2013; 7: 333-337. 
9. Bakri YN, Ezzat A, Akhtar, Dohami, Zahrani. Malignant germ cell tumors of the ovary. Pregnancy considerations. Eur J Obstet Gynecol Reprod Biol 2000; 90: 87-91.

10. Hasdemir P.S, Guvenal T, Menekse S, Solmaz U, Kandiloglu A.R, Koyuncu F.M, et al. Ovarian Immature Teratoma Detected During Pregnancy. Journal of Medical Science and Discovery 2016; 3(1): 1-6

11. Gershenson DM, del Junco G, Silva EG, Copeland LJ, Wharton JT, Rutledge FN. Immature teratoma of the ovary. Obstet Gynecol 1986; 68: 624-9.

12. Han JY, Nava-Ocampo AA, Kim TJ, Shim JU, Park CT. Pregnancy outcome after prenatal exposure to bleomycin, etoposide and cisplatin for malignant ovarian germ cell tumors: report of 2 cases. Reprod Toxicol 2005; 19: 557-61.

13. Elit L, Bocking A, Kenyon C, Natale R. An endodermal sinus tumor diagnosed in pregnancy: case report and review of the literature. Gynecol Oncol 1999; 72: 123-7.

14. Poremba C, Dockhorn-Dworniczak B, Merritt V, Li CY, Heidl G, Tauber PF, et al. Immature teratomas of different origin carried by a pregnant mother and her fetus. Diagn Mol Pathol 1993; 2: 131-6

Figure legends:

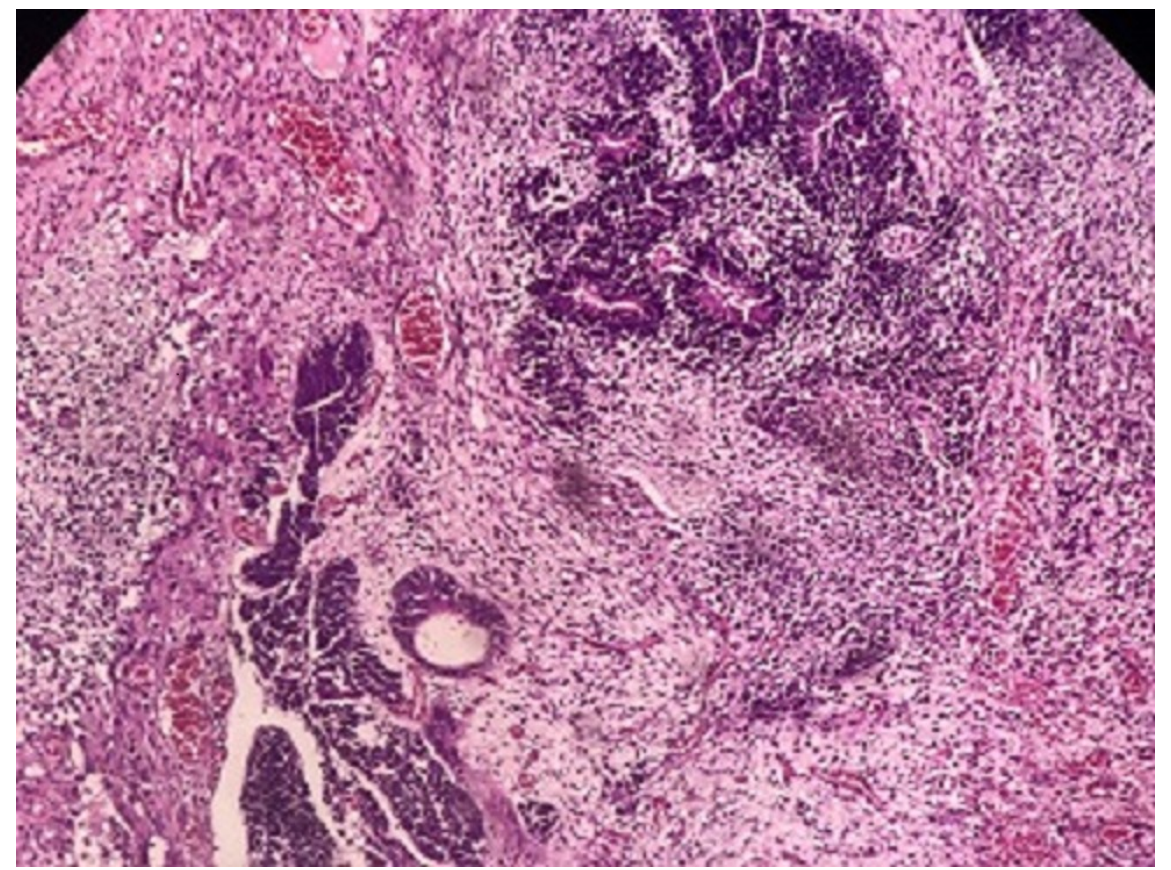

Figure 1: Immature component of teratoma including neuroepithelial elements with rosette formation 


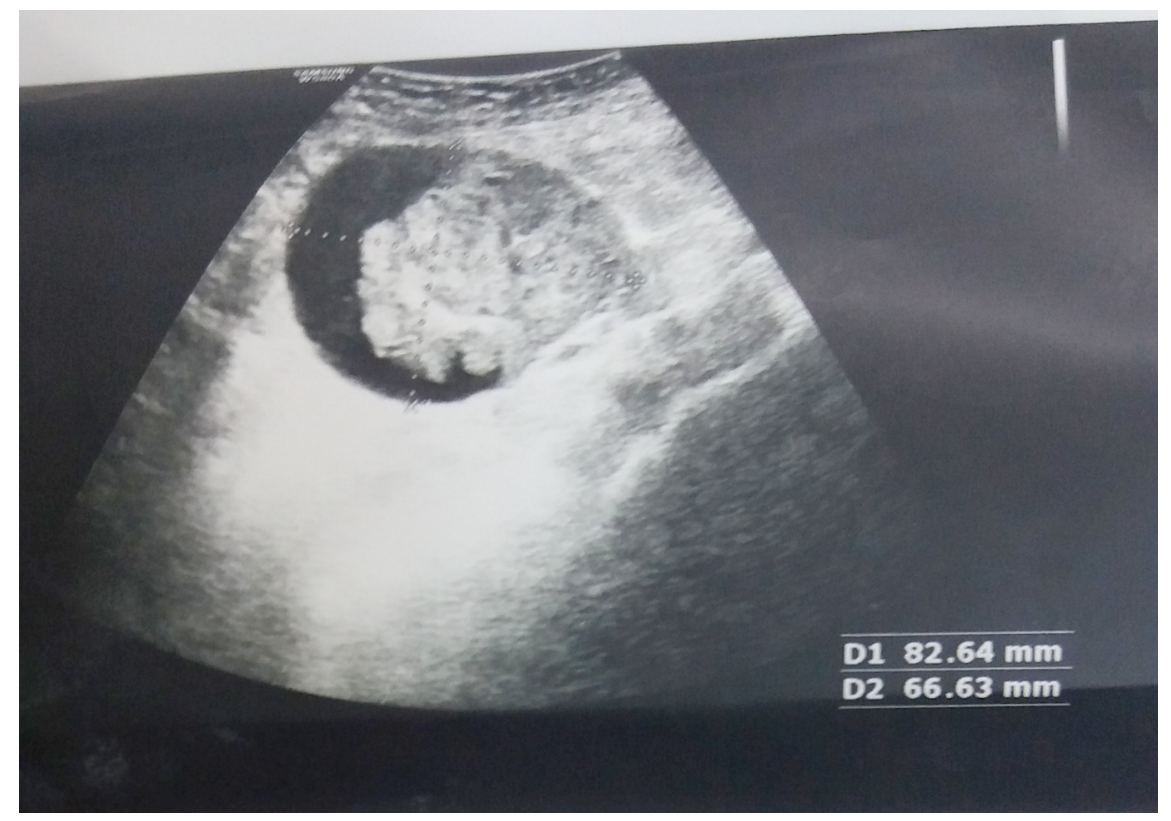

Figure 2: Ovarian cystic mass with solid component

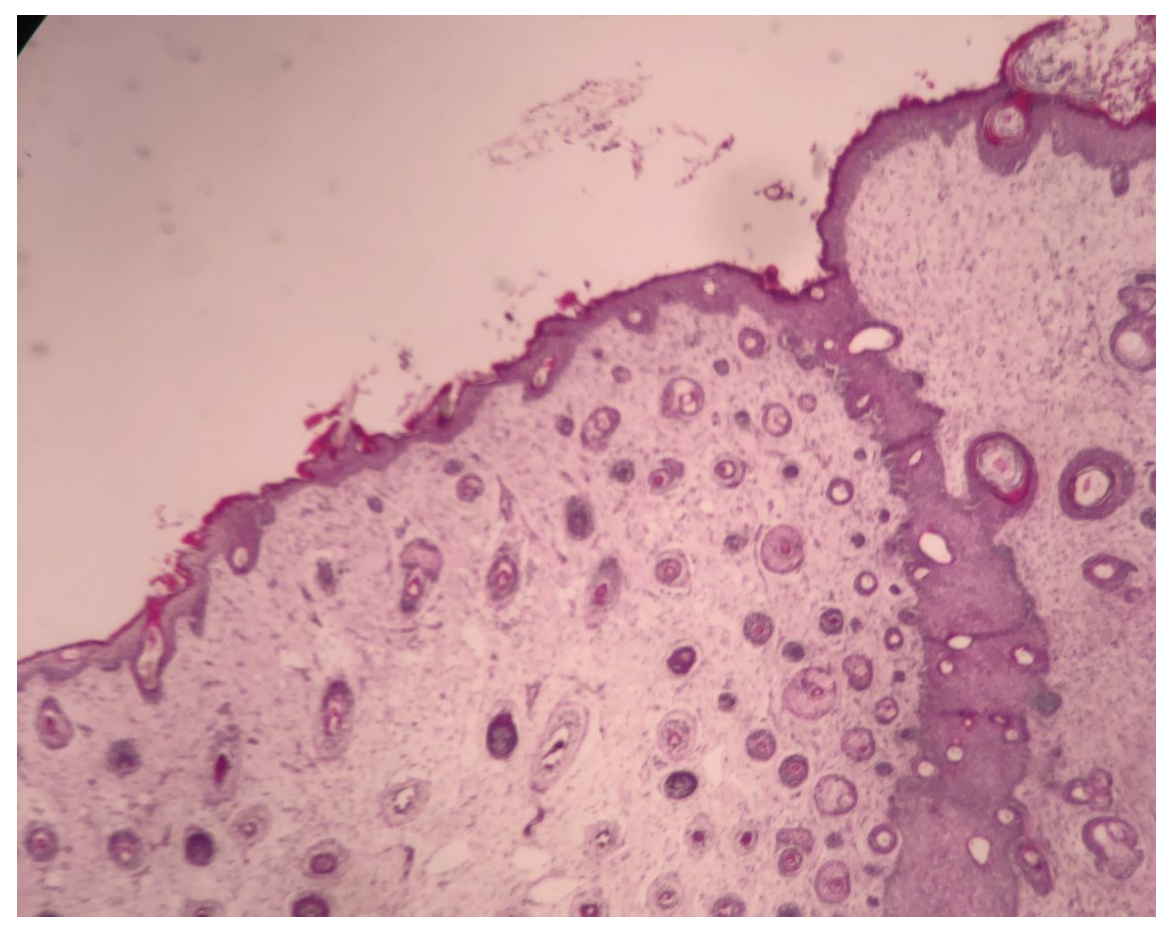

Figure 3: Mature ectodermal component including squamous epithelium and hair follicles 